\title{
MODELLING OF THE BEHAVIOUR OF ARAMID FOLDED CORES UP TO GLOBAL CRUSHING
}

\author{
E. Baranger, C. Cluzel, P.-A. Guidault, O. Allix \\ LMT-Cachan (ENS Cachan/CNRS/UPMC/PRES UniverSud Paris) \\ 61 av. du Président Wilson, F-94230 Cachan, France \\ \{baranger,cluzel,guidault,allix $\} @$ lmt.ens-cachan.fr
}

\begin{abstract}
SUMMARY
This paper presents the basic ingredients needed to model the out-of-plane compaction behaviour of an aramid paper folded core up to the global collapse load. The proposed approach is both experimental and numerical. Different scales are taken into account: fiber, paper and core scales. The influence of geometrical defects is demonstrated.
\end{abstract}

Keywords: , folded core, geometrical defects, sandwich, aramid paper, crushing.

\section{INTRODUCTION}

Sandwich structures are good candidates for the aeronautical industry as they provide a good stiffness/mass ratio. Aramid paper honeycomb cores are one of the best candidates. Nevertheless, it is not used for primary structures applications on airplanes. The aim of the CELPACT European project (CELlular structures for impact performance) is to develop new core materials for this kind of application. One of these materials is an aramid paper folded core developed by $[1,2]$. In order to give to the aeronautical industry the keys to choose this kind of material, a complete range of studies has been performed, from experimental observations to validation via numerical modelling.

An extensive work has been done on the behaviour of honeycomb cores with both experimental and numerical approaches. It mainly focuses on three points: elastic properties, failure criterion and energy absorption. Most of the work couple both experimental and modelling aspects. For example, in [3], an analytical study of the out of plane crushing as well as shearing of a unit cell is compared to experimental results. Two failure criterion are given: the bucking of a cell and the failure of the cell walls. In $[4,5]$, the in-plane biaxial crushing of a core is studied experimentally and compared to finite element analyses. The authors show a good correlation between simulations and experiments on the global stress/displacement curves. More recently, [6] used periodic homogenisation to get the elastic properties of honeycombs and also defined a failure criterion based on a linear buckling analysis. A good physical understanding of the crushing of honeycomb cores is given in [7] which also gives a macro model of the core. 
Concerning folded cores, mainly the manufacturing process has been treated in $[1,8]$. A wide range of experimental results have been conducted and are presented in [9] or [10]. Numerical models have been set up mainly using explicit codes to be able to go through instabilities $[11,12]$.

The aim of this paper is to give the basic physical key points in order to have a predictive modelling of the behaviour of an aramid folded core under compaction (up to the global instability load). The influence of geometrical defects for such kind of cores is emphasised. Note that honeycomb cores don't seem to exhibit such defect dependency. A multiscale study has been performed. The different scales are:

- The microscale: scale of the aramid fiber;

- The mesoscale: scale of the paper;

- The macroscale: scale of the core.

From observations at the microscale, the paper is modelled as a three-layer laminate. The inner layer is rich in fibers and matrix, with a quasi-constant thickness while the outer layers are only made of matrix with a highly variable thickness.

At the mesoscale, the multilayer laminate model has been completed with a first basic damage evolution law and identified. For that, tension and bending tests on paper coupons have been performed. For bending tests, buckling loadings techniques have been used on.

At the macroscale, a global crushing test has been performed on a core. It leads to the observation of the main degradation mechanisms and to the global force/displacement curve of the core. By using the damage model described above for the paper, a numerical model of the core coupon has been developed and numerically tested. It is shown that, for a given folding pattern, defects play a major role in the degradation mechanisms of the core up to the peak load. Thus, a manufacturing defect (observed on the real cores) is generated by simulation of the folding process of the core from a paper sheet.

First numerical results are in good agreement with experimental results up to the global instability load where other degradation mechanisms have to be modelled as the creation of new folds and tears for example.

This paper is divided into three parts. The first part introduces the core and the different scales. The second and third parts focus on two key points: the paper modelling and the geometrical defects modelling. The third part also presents experimental results on global crushing.

\section{SANDWICH DESCRIPTION AND SCALES DEFINITIONS}

The studied core is made of N637 paper from Dupon De Nemour. This paper is a mat of aramid fibers in a phenolic matrix (figure 2a). The core is obtained from sheets of prepreg paper by a continuous folding process ended by phenolic matrix polymerization at elevated temperature [2]. In order to create a folding pattern, some marks are made on the paper sheet either by cutting or rolling. The folded core geometry is presented on figure 1 (thickness of the core: $20 \mathrm{~mm}$, thickness of the paper: $0.3 \mathrm{~mm}$ ). A nominal unit 
cell is also presented. Note that geometrical defects can be observed on figure 1, particularly at the edges of the sample.

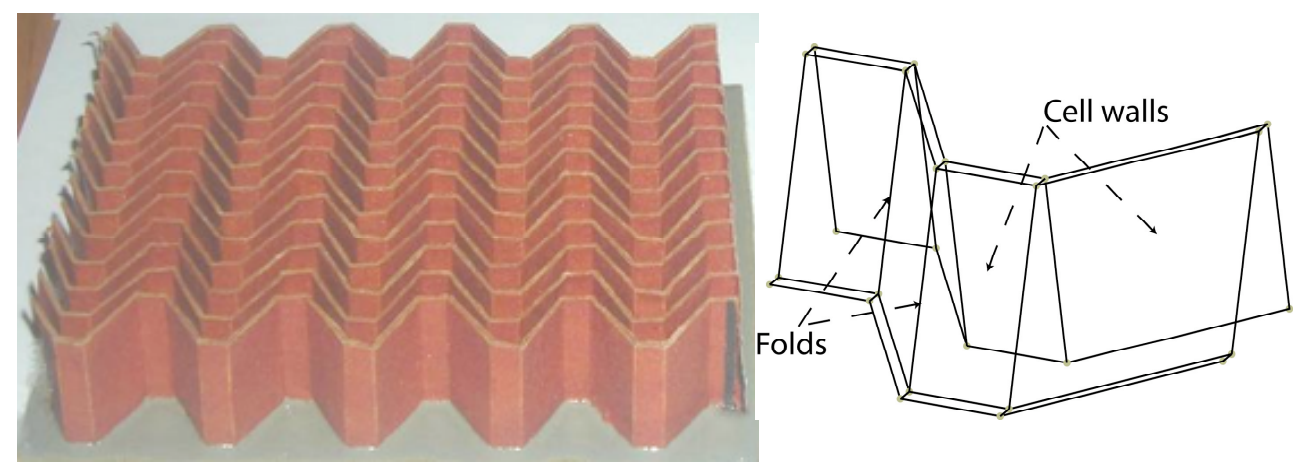

Figure 1: Folded core and nominal unit cell

Different scales are considered in this study. The scale of the aramid fiber is called microscale, its characteristic length is about $0.01 \mathrm{~mm}$ (diameter of the aramid fibers). The scale of the paper is called mesoscale, its characteristic length is about $0.3 \mathrm{~mm}$ (paper thickness). The scale of the core is called macroscale, its characteristic length is about $20 \mathrm{~mm}$ (core thickness and folding pattern size). The scale of the sandwich panel that mainly interest engineers for structure computations will not be considered in the following.

\section{PAPER MODELLING}

At the microscale (only the paper is studied), two components are visible: the aramid fiber and the phenolic matrix. Samples of paper sheet have been embedded in resin and polished to be able to observe their microstructure by using optical or SEM microscopy techniques.

Three different layers can be distinguished through the paper thickness on figure $2 \mathrm{a}$, where two paper sheets are observed. The inner one is mainly composed of aramid fibers and matrix, its thickness is almost constant (about $0.2 \mathrm{~mm}$ ). The outer layers are only composed of matrix and their thickness is highly variable. Fibers seem to be oriented almost randomly. Porosity and lack of matrix can also be seen, for both the inner and the outer layers. The overall thickness is very difficult to measure directly from micrographics, thus it has been measured using a standard mechanical micrometer and is estimated about $0.33 \mathrm{~mm}$. 

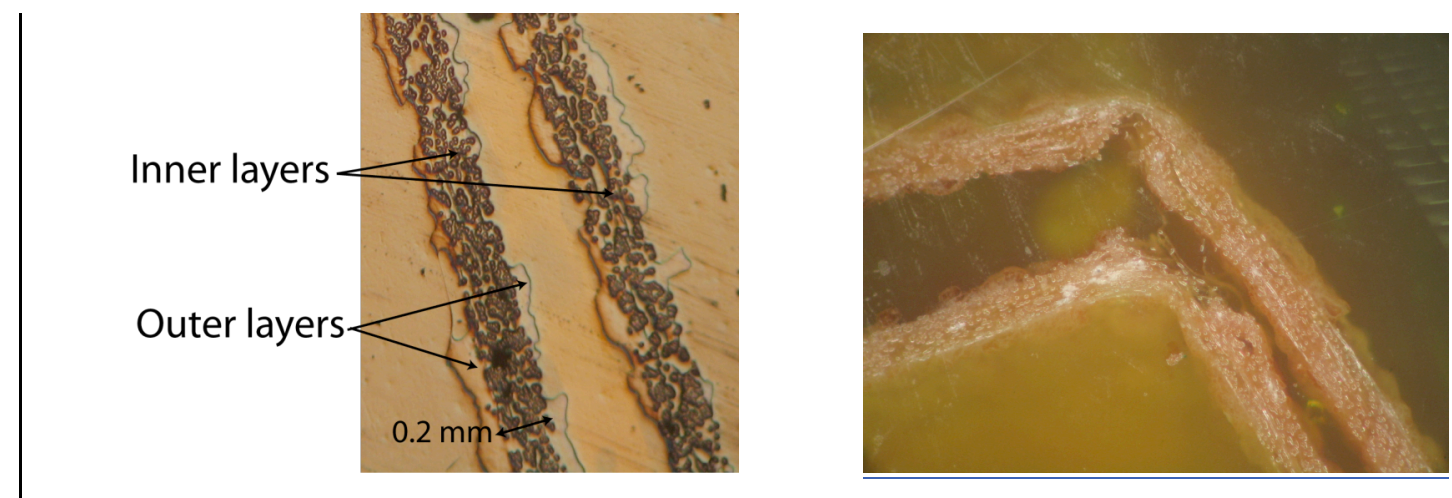

Figure 2: Through thickness observation of two aramid paper sheets (a) and cross section of two "vertical" folds (b)

In order to observe the degradation scenario occurring during the bending (or folding) of an originally virgin paper sheet, a simple buckling test has been performed and observed using SEM. The scenario is the following:

1. creation of cracks in the outer layers;

2. propagation of the cracks through the inner layer;

3. debonding at the fiber/matrix interphase;

4. breakage of the fibers.

Considering the observations above, the constitution of the paper as well as its degradation mechanisms are related to the three layers structure composing a sheet of paper. As a consequence, the paper will be modelled as a three layers laminate. The inner one is supposed of constant thickness of $0.2 \mathrm{~mm}$. The outer ones are supposed to be made of phenolic matrix only (Young's modulus $3000 \mathrm{MPa}$, Poisson's ration 0.4). The elastic properties of the inner layer and the thickness of the outer layers are identified by both a tensile test and a vibration bending test where the first free vibration frequency is used to get the bending behaviour of the coupon.

Considering the non-linear behaviour of the paper, different tests have been performed. In tension, the paper exhibits a non-linear behaviour that is different in the transverse and longitudinal directions (see figure 3). Note that, due to the folding pattern, the longitudinal direction mainly contributes to the compressive behaviour of the core. Furthermore, in the sandwich structure, the paper cell walls are mainly loaded in compression and bending. Thus the non-linear evolutions in tension may not be sufficient and are completed by a bending test performed in the longitudinal direction using an Euler buckling experimental setup. Despite the anelastic strain observed on figure 3, a first basic damage model is chosen to describe the envelope of the force/displacement curve. Each layer has its own damage variable, which is assumed to evolve linearly according to the strain when a strain threshold is reached. This kind of behaviour is able to represent correctly the observed behaviour in bending and in tension in the longitudinal direction. 


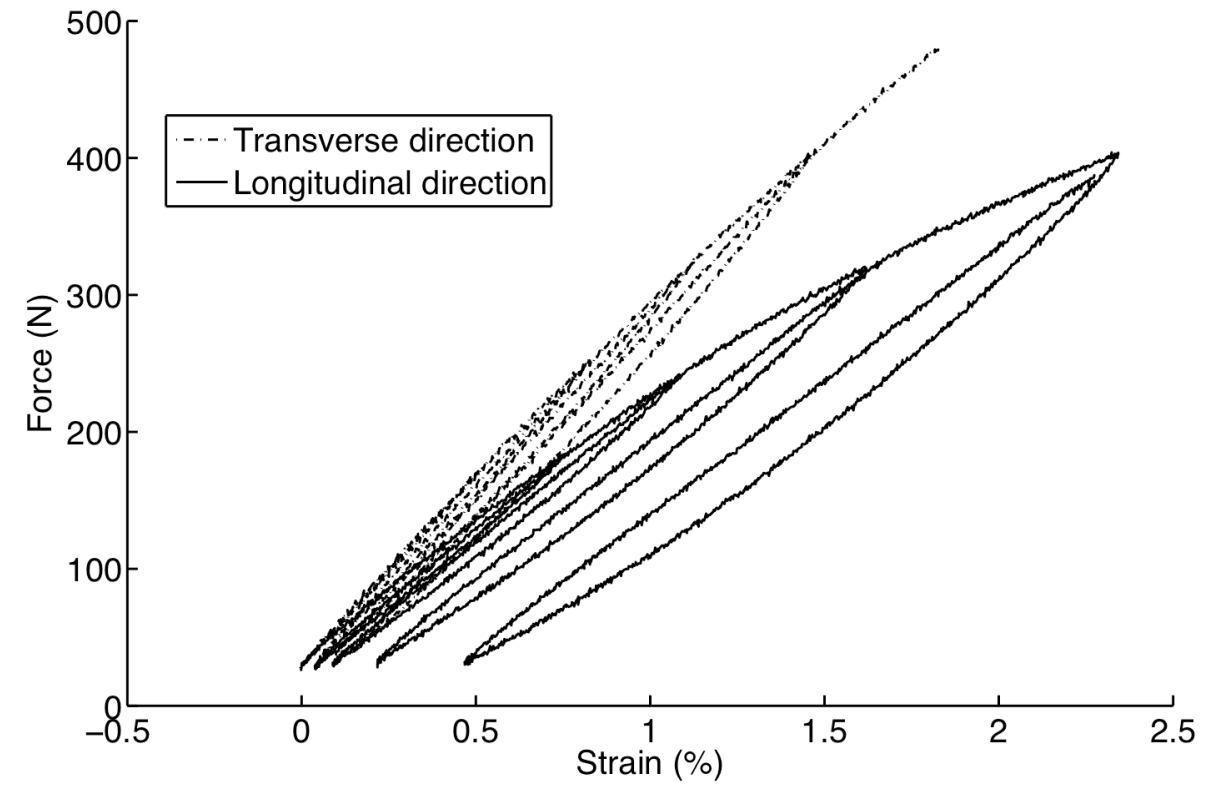

Figure 3: Tension behaviour of a paper coupon in longitudinal and transverse directions

\section{GLOBAL CRUSHING AND GEOMETRICAL DEFECTS MODELLING}

A compaction test has been performed up to the total crushing of a sandwich. A hinge has been placed on one side of the sandwich in order to load it properly. The relative displacement of the two skins of the sandwich has been measure using a LVDT sensor. Front and back faces of the sample have been observed using two digital cameras. The displacement velocity is $0.1 \mathrm{~mm} / \mathrm{min}$. The core is composed of $5 \times 13$ elementary cells and the sample dimensions are $120 \times 107 \times 24 \mathrm{~mm}^{3}$. On figure 4, the continuous fine black line represents the measured data during the test while the continuous coarse black line represents the experimental data corrected by taking into account the stiffness of the experimental setup (hinge, ...) . 


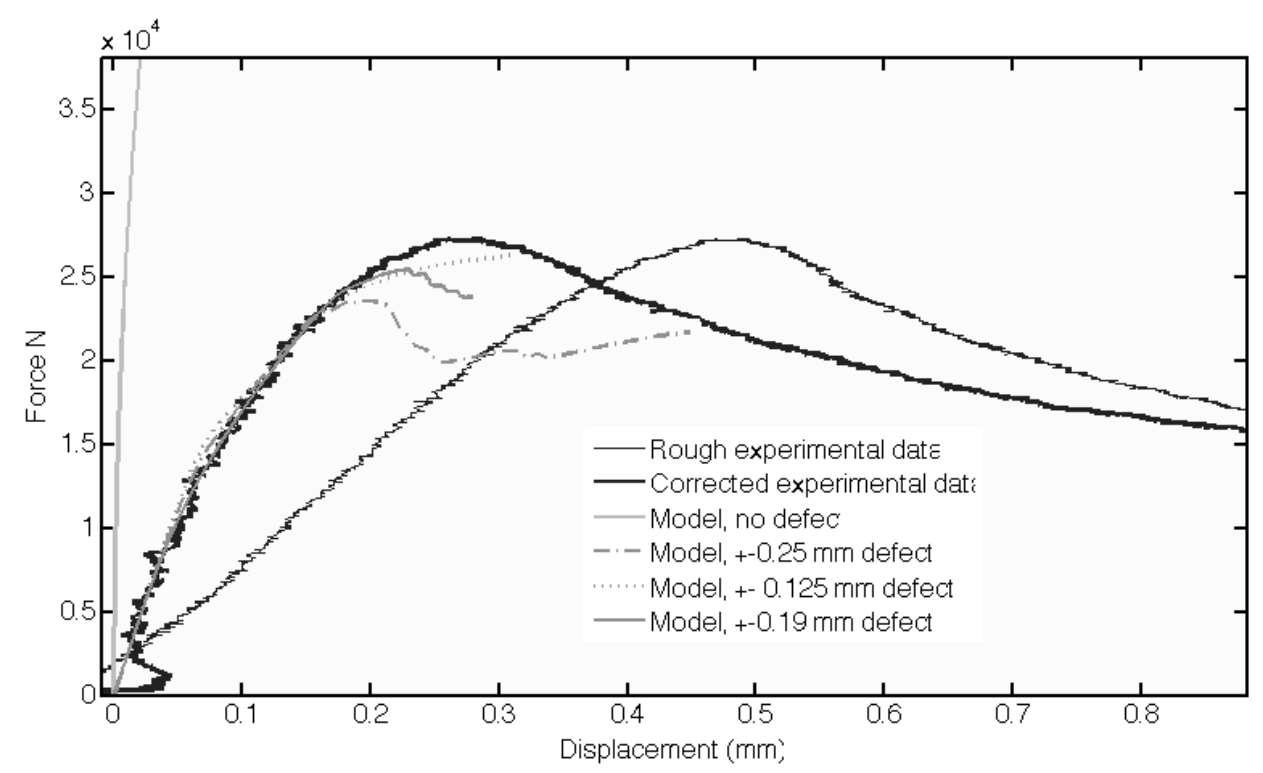

Figure 4: Force / displacement curve of a core compression test

On figure 5, an observation of the core is presented for a load just after the global limit load. In this configuration, tears or new folds have not yet appeared. Displacements are quite important. Strains are relatively small but damage already occurred in the external layers of the paper.

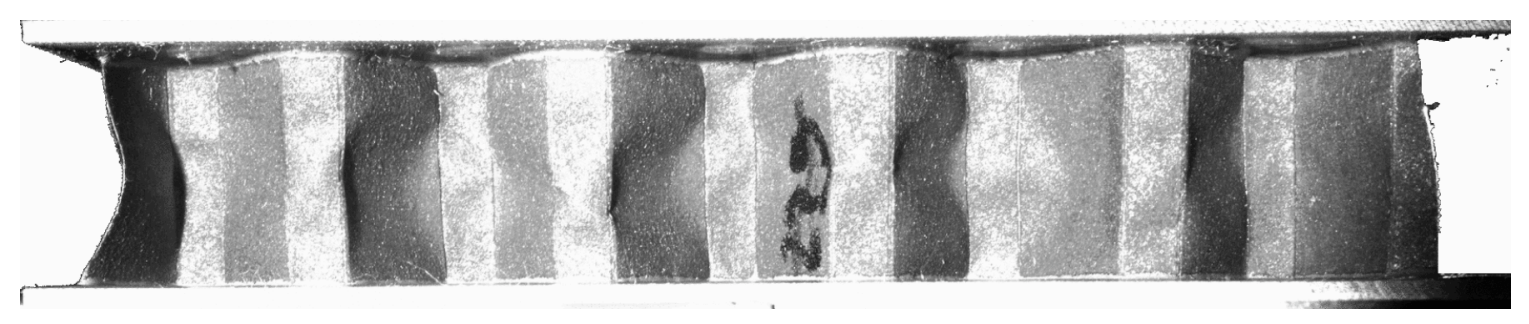

Figure 5: Core observation at the global limit load

A numerical implicit quasi-static analysis as been conducted with Abaqus standard. As the problem to solve is a stability problem, the geometrical defects may have a strong influence on the results [13].

First, a perturbed geometry of the core has been created by an elastic simulation of the folding process. On figure 6 , the nominal and perturbed folding patterns for a unit cell are depicted before folding. The locations of folds are modeled using perfect hinges on a plane sheet of paper. In order to generate a defect, each vertex of the folding pattern is perturbed randomly (on figure 6, only one vertex is perturbed). This perturbation can be seen as an approximate folding scenario of the core during the folding process. The folding process is simulated for 3 lines of 5 cells per line (figure 7). One clearly notices that the perturbed geometry leads to significant defects on the folded geometry. 

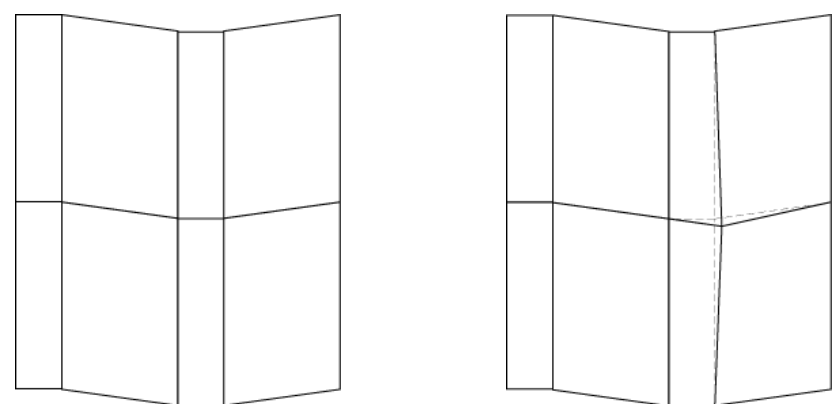

Figure 6: Nominal and perturbed unit cell geometry before folding

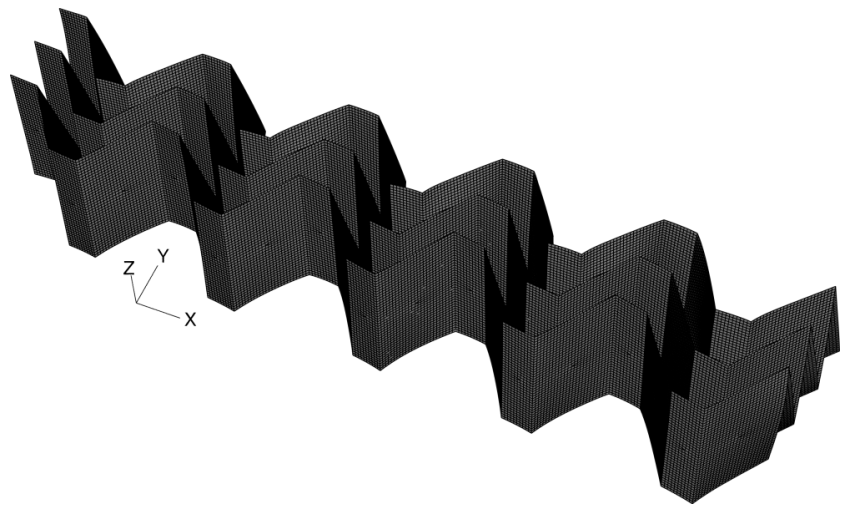

Figure 7: Perturbed folded geometry

Second, the crushing simulation is performed on this geometry using the damageable behavior identified above. Only the middle line of five cells is retained in order to avoid non-physical geometrical defects due to improper boundary condition on the two other lines generated during the folding process simulation. The lower face of the simulated core is clamped and a pure vertical displacement is imposed on the upper face of the core. Considering the very low thickness of a fold (figure 2b), the folds are still considered as perfect hinges. Self-contact is not taken into account. An implicit computation using Abaqus is performed.

Figure 8 shows the deformed shape and the evolution of the damage variable in an outer layer of the paper in the core just after the global critical load (for a displacement of $0.28 \mathrm{~mm})$. Dark zones correspond to high damage.

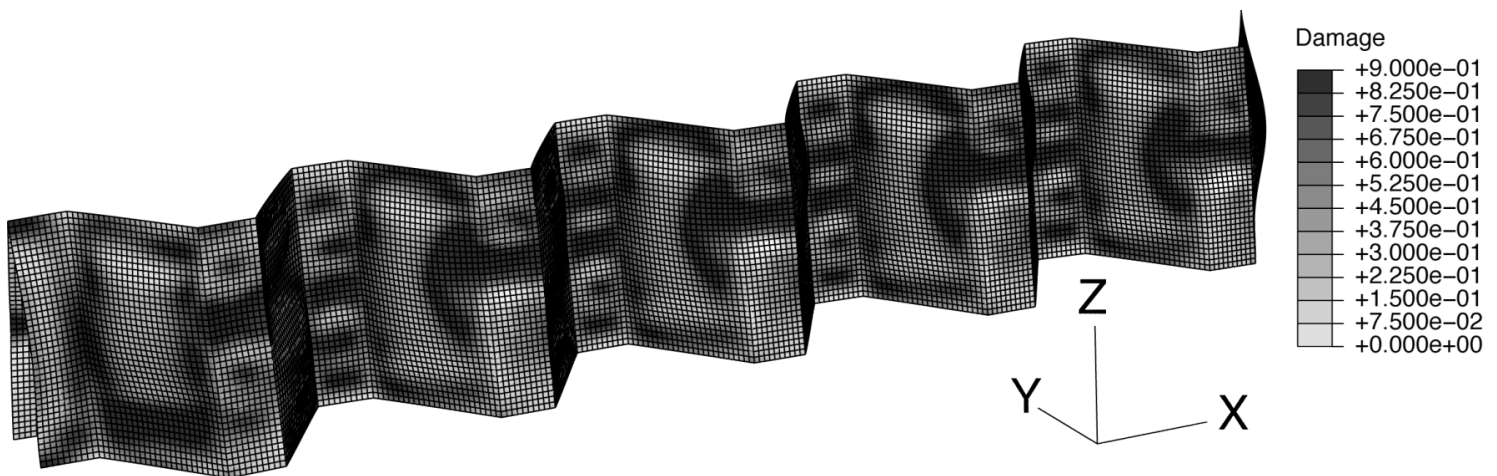

Figure 8: Deformed shape and damage field in the outer paper layer just after the critical load of a crushing test 
The associated results in term of global resulting force versus vertical displacement are plotted on figure 4 for different size of defects. The geometry before folding has been perturbed by a random value in the range of $\pm 0.125, \pm 0.19$ or $\pm 0.25 \mathrm{~mm}$ in the in-plane directions. This order of defect magnitude is in agreement with experimental observations. Let us note that modelling defects is essential according to figure 4; a model without defect leading (with the same material behavior) to a very stiff response. A defect of $\pm 0.19 \mathrm{~mm}$ leads to a very good agreement between the simulation and the experimental results. The shape of the deformed configuration is also in good agreement with the experimental one (figure 5 and figure 8).

Note that other less physical defects (first buckling modes on the nominal geometry for example) have been tested and don't lead to such good results.

\section{CONCLUSION}

This paper presents the basic ingredients needed to model the compaction behaviour of an aramid paper folded core up to the global collapse load under out-of-plane compression. The proposed approach is both experimental and numerical. It also takes into account the available information at different scales.

Two main points have been studied. A first modelling of the paper behaviour has been proposed and identified. It is a laminate like model based on micro observations of the paper. The behaviour of each layer of the laminate is modelled using a simple isotropic damageable law. These laws are identified in the longitudinal direction using tensile and bending experimental results. The second point concerns the modelling of defects. As the crushing of the folded core is a stability problem, geometrical defects play an essential role. The geometrical folded configuration is generated by simulation of the folding process. For that, a nominal folding pattern on a plane paper sheet is perturbed. The folding is then computed with an implicit Abaqus simulation. The predicted deformed shape is in good agreement with the experimental results.

\section{ACKNOWLEDGEMENTS}

The E.U. is greatly acknowledged for its funding of the CELPACT European project, the presented work is part of this project.

\section{References}

1. DRECHSLER K, KEHRLE R. Manufacturing of Folded Core-Structures for Technical Applications. 25th International SAMPE Europe Conference, Paris, 2004, pp. 508-513.

2. DRECHSLER K, KEHRLE R, KLETT Y, THORSTEINSSON E. Faltwabenkerne für multifunktionale Leichtbaustrukturen. 2. Materialica Composites-Kongress "Composites in Automotive \& Aerospace", München, 2006. 
3. ZHANG J, ASHBY M F. The out-of-plane properties of honeycombs. Int. J. Mech. Sci., 1992, 34(6), p. 475-489.

4. PAPKA S, KYRIAKIDES S. Biaxial crushing of honeycombs - Part I: Experiments. Int. J. Sol. Struct. 1999, 36, p 4367-4396.

5. PAPKA S, KYRIAKIDES S. Biaxial crushing of honeycombs - Part II: Analysis. Int. J. Sol. Struct. 1999, 36, p 4397-4423.

6. GORNET L, MARCKMANN G, LOMBARD M. Détermination des coefficients d'élasticité et de rupture d'âmes nids d'abeilles Nomex : homogénisation périodique et simulation numérique. Mécanique et Industries, 2006, 6, p. 595-604.

7. AMINANDA Y, CASTANIE B, BARRAU J-J, THEVENET P. Experimental analysis and modelling of the crushing of honeycomb cores, Applied Composite Materials, 2005, 12, pp. 213-227.

8. ELSAYED E, BASILY B. A continuous folding process for sheet materials. International Journal of Materials and Product Technology, 2004, 21(1/2/3), p. 217- 238 .

9. KINTSCHER M, KÄRGER L, WETZEL A, HARTUNG D. Stiffness and failure behaviour of folded sandwich cores under combined transverse shear and compression. Composites Part A, 2007, 38, p. 1288-1295.

10. FISCHER S, DRECHSLER K, KILCHERT S, JOHNSON A. Mechanical Tests for FoldCore Base Material Properties. CompTest 2008, Dayton, 2008.

11. NGUYEN M, JACOMBS S, THOMSON R, HACHENBERG D, SCOTT M. Simulation of impact on sandwich structures. Composite structures, 2005, 67, p. 217-227.

12. HEIMBS S. Virtual testing of sandwich core structures using dynamic finite element simulations. Computational Materials Science, 2009, 45, p. 205-216.

13. BAZANT Z, CEDOLIN L. Stability of Structures: Elastic, Inelastic, Fracture and Damage Theories. New York: Oxford University Press, 1991, 1011 pages. ISBN 0-486-42568-1. 